\title{
Preparation, Microwave Transitions and Dipole Moment of
}

\section{trans-1-Fluoro-1,3-butadiene}

\author{
PER CEDERBALK
}

Department of Physical Chemistry, Arrhenius Laboratory, University of Stockholm, S-10691 Stockholm, Sweden

Microwave transitions of trans-1-fluoro-1,3-butadiene have been recorded and measured in the region $18000-26500 \mathrm{MHz}$. The ground vibrational state rotational constants found were $A_{0}=34371$ $\pm 18 \mathrm{MHz}, B_{0}=2170.669 \pm 0.001 \mathrm{MHz}$ and $C_{0}=$ $2041.682 \pm 0.001 \mathrm{MHz}$. The fundamental frequency of the lowest-energy normal mode which is a torsion was estimated from the intensity of the vibrational satellites. The value obtained was $138 \pm 11 \mathrm{~cm}^{-1}$. The total dipole moment was found to be $1.380 \pm$ $0.017 \mathrm{D}$ from a study of the Stark effect.

\section{EXPERIMENTAL}

The preparation of trans-1-fluoro-1,3-butadiene has been described by Viehe et al. ${ }^{1-3}$

$$
\begin{aligned}
& \mathrm{CHBr}_{2}-\mathrm{CHBr}_{2} \stackrel{\mathrm{SbF}_{3}, \mathrm{Br}_{2}}{\longrightarrow} \mathrm{CHBrF}-\mathrm{CHBr}_{2} \\
& \mathrm{CHBrF}-\mathrm{CHBr}_{2} \stackrel{\text { Zn,ethanol }}{\longrightarrow} \mathrm{CHF}=\mathrm{CHBr} \\
& \mathrm{CHF}=\mathrm{CHBr} \stackrel{\mathrm{I}_{2}, \mathrm{NaI}}{\longrightarrow} \mathrm{CHF}=\mathrm{CHI} \\
& \mathrm{CHF}=\mathrm{CHI}+\mathrm{CH}_{2}=\mathrm{CH}_{2} \stackrel{h v}{\rightarrow} \\
& \mathrm{CHF}=\mathrm{CH}-\mathrm{CH}_{2}-\mathrm{CH}_{2} \mathrm{I} \\
& \mathrm{CHF}=\mathrm{CH}-\mathrm{CH}_{2}-\mathrm{CH}_{2} \mathrm{I} \stackrel{\mathrm{KOH}, 1-\text { butanol }}{\rightarrow} \\
& \mathrm{CHF}=\mathrm{CH}-\mathrm{CH}_{=}=\mathrm{CH}_{2}
\end{aligned}
$$

The recommended reaction temperature, $250{ }^{\circ} \mathrm{C}$, turned out to be too high for the iodine-bromine exchange (III). The reaction was successfully carried out, however, at temperatures between 150 and $175^{\circ} \mathrm{C}$. The sodium iodide had been dried in vacuum, and likewise carefully dried 2-fluoro-1bromoethene (calcium chloride and subsequently molecular sieve $4 \AA$ ) was used for this reaction. Reaction (III) was run in an evacuated ampoule placed in a steel bomb which also contained some water, so that the high pressure within the ampoule during the reaction was partly compensated for by the vapour pressure of the water. In this way a reaction time of about $20 \mathrm{~h}$ could be achieved with an intact ampoule.

1-Fluoro-4-iodo-1-butene was dehydroiodated with potassium hydroxide in 1-butanol at $0{ }^{\circ} \mathrm{C}(\mathrm{V})$. The product 1-fluoro-1,3-butadiene was isolated by vacuum distillation and identified with infrared spectroscopy. The cis- and trans-isomers of the product were separated with gas-liquid chromatography at room temperature $\left(25^{\circ} \mathrm{C}\right)$. The column, with a length of $6 \mathrm{~m}$ and a diameter of $10 \mathrm{~mm}$, was packed with diethylhexylsebacate $(20 \%)$ absorbed on Chromosorb. A small amount of hydroquinone was added to prevent polymerization, and the samples were stored at $-20^{\circ} \mathrm{C}$.

The microwave spectra were recorded on a Hewlett-Packard Model 8460A microwave spectrometer with Stark modulation at $33.33 \mathrm{kHz}$. The measurements were made in the $\mathrm{K}$-band region $(18000-26500 \mathrm{MHz})$, at about $-20^{\circ} \mathrm{C}$ and at a pressure of $10 \mathrm{mT}$ Torr. The Stark lobes were recorded at pressures ranging from 18 to 24 mTorr. The uncertainty in the measured frequencies was estimated to be $0.03 \mathrm{MHz}$ (sweep rate $0.02 \mathrm{MHz} / \mathrm{s}$ ) for the lines and $0.05 \mathrm{MHz}$ (sweep rate $0.05 \mathrm{MHz} / \mathrm{s}$ ) for the Stark lobes.

\section{MICROWAVE TRANSITIONS AND ROTATIONAL CONSTANTS}

trans-1-Fluoro-1,3-butadiene was expected to be an almost symmetric prolate rotor, with both $\mu_{\mathrm{a}}$ and $\mu_{\mathrm{b}}$ dipole moment components. From the 


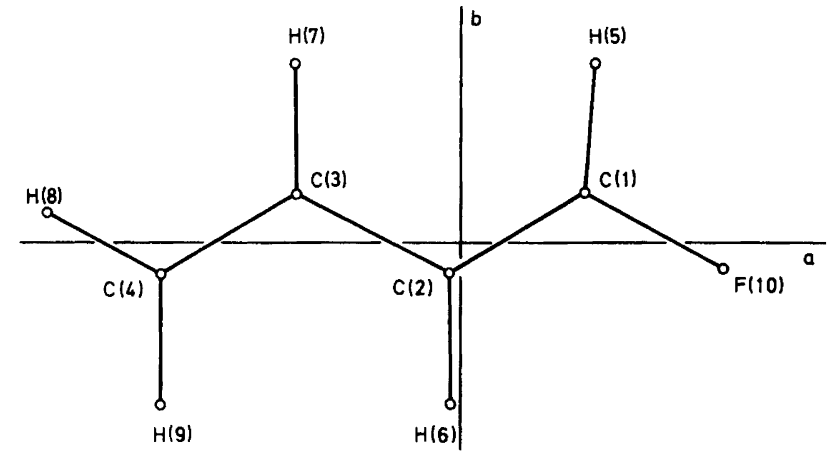

Fig. 1. Assumed structure of trans-1-fluoro-1,3-butadiene with principal axes of inertia. Values of the assumed structural parameters are given in Table 1.

Table 1. Assumed structural parameters for trans1-fluoro-1,3-butadiene.

\begin{tabular}{llll}
\hline \multicolumn{2}{l}{ Bond length $(\AA)$} & \multicolumn{2}{l}{ Angle ( $\left.{ }^{\circ}\right)$} \\
\hline $\mathrm{C}(1)=\mathrm{C}(2)$ & 1.33 & $\mathrm{C}(1)=\mathrm{C}(2)-\mathrm{C}(3)$ & 122 \\
$\mathrm{C}(2)-\mathrm{C}(3)$ & 1.46 & $\mathrm{C}(2)-\mathrm{C}(3)=\mathrm{C}(4)$ & 122 \\
$\mathrm{C}(3)=\mathrm{C}(4)$ & 1.34 & $\mathrm{H}(5)-\mathrm{C}(1)=\mathrm{C}(2)$ & 125 \\
$\mathrm{C}(1)-\mathrm{F}(10)$ & 1.35 & $\mathrm{~F}(10)-\mathrm{C}(1)=\mathrm{C}(2)$ & 121 \\
all C-H & 1.10 & $\mathrm{C}(1)=\mathrm{C}(2)-\mathrm{H}(6)$ & 120 \\
& & $\mathrm{H}(7)-\mathrm{C}(3)=\mathrm{C}(4)$ & 120 \\
& & $\mathrm{C}(3)=\mathrm{C}(4)-\mathrm{H}(8)$ & 121 \\
& & $\mathrm{C}(3)=\mathrm{C}(4)-\mathrm{H}(9)$ & 121 \\
\hline
\end{tabular}

assumed structure (obtained mainly from the structure of cis-1-chloro-1,3-butadiene; to be published) given in Fig. 1 and Table 1 the rotational constants $A, B$ and $C$ and the asymmetry parameter $\kappa$ were calculated. The estimated values were: $A=33197, B=2181.2$ and $C=2046.7 \mathrm{MHz}$ and consequently $\kappa=-0.99136$. Because of the value of $\kappa$ the lines of the spectrum were expected to appear in bands as is typical for an almost symmetric prolate rotor molecule. This was confirmed, and the $a$-type $R$-branch transitions were quite easily found in the spectrum. The measured frequencies are given in Table 2. (The $a$-type $R$ branch transitions having $K_{-1}>2$ were not possible to resolve from each other, and therefore they were not measured.) The measured frequencies were used in a least-squares fit to calculate the rotational constants and the centrifugal distortion constants $\Delta_{\mathrm{J}}$ and $\Delta_{\mathrm{JK}}$. It was not possible to get accurate values for the centrifugal distortion constants $\Delta_{\mathrm{K}}$, $\delta_{\mathrm{J}}$ and $\delta_{\mathrm{K}}$ due to the fact that only $a$-type $R$-branch transitions were found. This had the further con-

Table 2. Observed and calculated ${ }^{a}$ rotational transition frequencies $(\mathrm{MHz})$ in the ground and excited states of trans-1-fluoro-1,3-butadiene.

\begin{tabular}{llclrlr}
\hline Transition & $\begin{array}{l}v=0 \\
\text { Obs }\end{array}$ & Obs-Calc & $\begin{array}{l}v=1 \\
\text { Obs }\end{array}$ & Obs-Calc & \multicolumn{1}{l}{$\begin{array}{l}\text { Obs } \\
\text { Obs-Calc }\end{array}$} & Obs- \\
\hline $5_{15} \leftarrow 4_{14}$ & 20737.585 & 0.004 & 20779.568 & 0.006 & 20821.461 & -0.040 \\
$5_{05} \leftarrow 4_{04}$ & 21053.948 & 0.007 & 21088.479 & -0.008 & 21123.084 & 0.029 \\
$5_{24} \leftarrow 4_{23}$ & 21061.058 & 0.010 & 21095.648 & 0.018 & 21130.625 & -0.017 \\
$5_{23} \leftarrow 4_{22}$ & 21068.774 & -0.007 & 21103.451 & -0.017 & 21138.473 & 0.011 \\
$5_{14} \leftarrow 4_{13}$ & 21382.506 & 0.005 & 21409.754 & 0.008 & 21437.155 & 0.010 \\
$6_{16} \leftarrow 5_{15}$ & 24884.012 & 0.009 & 24934.366 & 0.018 & 24984.662 & -0.010 \\
$6_{06} \leftarrow 5_{05}$ & 25260.434 & -0.002 & 25301.799 & -0.016 & 25343.332 & 0.030 \\
$6_{25} \leftarrow 5_{24}$ & 25272.510 & 0.004 & 25313.978 & 0.001 & 25355.978 & -0.010 \\
$6_{24} \leftarrow 5_{23}$ & 25286.044 & 0.006 & 25327.684 & -0.007 & 25369.699 & 0.029 \\
$6_{15} \leftarrow 5_{14}$ & 25657.893 & 0.009 & 25690.562 & 0.017 & 25723.366 & -0.055 \\
\hline
\end{tabular}

${ }^{a}$ To calculate frequencies the molecular constants of Table 3 were used. 
Table 3. Rotational constants $(\mathrm{MHz})$, principal moments of inertia ${ }^{a}\left(u \AA^{2}\right)$, inertial defect $\left(u \AA^{2}\right)$ and centrifugal distortion constants $(\mathrm{kHz})$ for the ground and excited states of trans-1-fluoro-1,3-butadiene.

\begin{tabular}{llll}
\hline & $v=0$ & $v=1$ & $v=2$ \\
\hline$A$ & $34371(18)$ & $32507(42)$ & $31191(87)$ \\
$B$ & $2170.669(1)$ & $2172.664(4)$ & $2174.666(8)$ \\
$C$ & $2041.682(1)$ & $2046.624(4)$ & $2051.534(8)$ \\
& & & \\
$I_{\mathrm{a}}$ & $14.704(8)$ & $15.547(20)$ & $16.203(45)$ \\
$I_{\mathrm{b}}$ & $232.8204(1)$ & $232.6066(4)$ & $232.3925(9)$ \\
$I_{\mathrm{c}}$ & $247.5292(1)$ & $246.9315(5)$ & $246.3405(10)$ \\
$\Delta^{b}$ & $0.005(8)$ & $-1.222(20)$ & $-2.255(45)$ \\
$\Delta_{\mathrm{J}}$ & $0.16(2)$ & $0.23(5)$ & $0.25(11)$ \\
$\Delta_{\mathrm{JK}}$ & $-5.8(2)$ & $-4.4(5)$ & $-15.9(11)$ \\
\hline
\end{tabular}

${ }^{a}$ The conversion factor used was $505376 \mathrm{MHz} u \AA^{2}{ }^{4}$

${ }^{b} \Delta=I_{\mathrm{c}}-I_{\mathrm{a}}-I_{\mathrm{b}}$.

sequence that the rotational constant $A$ could not be well determined: see Table 3 . In order to improve the accuracy of $A$ it would have been necessary to assign at least one of the $b$-type transitions. But because of the fact that the $b$-type transitions are much dependent on not only the rotational constant $A$ but also on the centrifugal distortion constants $\Delta_{\mathrm{K}}$ and $\delta_{\mathrm{K}}$, it was considered to be a very difficult task to find such transitions in this typical $a$-type spectrum. A couple of $b$-type $R$-branch transitions were sought, but were not found. From the value of the inertial defect in the ground state it was concluded that the molecule is planar and therefore has no $\mu_{\mathrm{c}}$ dipole moment component.

The spectrum has a prominent vibrational satellite structure. Four sequences with four or five lines in each could clearly be seen, and they were used to obtain the associated normal mode frequency assuming a Boltzmann distribution. The value obtained was $138 \pm 11 \mathrm{~cm}^{-1}$. This is concluded to be an out-of-plane motion as the inertial defect of the corresponding vibrational states decreases with increasing vibrational quantum number: see Table 3.

The fundamental vibrational frequencies were calculated from an assumed valence force field. The force constants were taken from a normal coordinate analysis, by Borg et al., ${ }^{5}$ of the vibrational spectrum of trans-1-chloro-1,3-butadiene,

Table 4. Measured displacements (MHz) of Stark lobes in the spectrum of trans-1-fluoro-1,3-butadiene.

\begin{tabular}{|c|c|c|c|c|c|c|}
\hline \multirow{2}{*}{$\begin{array}{l}\text { Transition } \\
5_{05} \leftarrow 4_{04}\end{array}$} & \multicolumn{2}{|c|}{$\begin{array}{l}\text { Electric field strength }(\mathrm{V} / \mathrm{cm}) \\
3147(3)\end{array}$} & \multicolumn{2}{|c|}{ 2573.1(4) } & \multicolumn{2}{|c|}{$2081.5(8)$} \\
\hline & $\begin{array}{r}|\mathbf{M}|=0 \\
1 \\
2\end{array}$ & $\begin{array}{l}-7.635 \\
-6.440 \\
-2.840\end{array}$ & $\begin{array}{l}1 \\
2\end{array}$ & $\begin{array}{l}-4.174^{a} \\
-1.810^{a}\end{array}$ & $\begin{array}{l}0 \\
1 \\
2\end{array}$ & $\begin{array}{l}-3.210 \\
-2.800 \\
-1.205\end{array}$ \\
\hline $5_{14} \leftarrow 4_{13}$ & $|\mathbf{M}|=0 \begin{array}{r}0 \\
1 \\
2 \\
3\end{array}$ & $\begin{array}{r}-2.910 \\
-7.395 \\
-20.360 \\
-40.435\end{array}$ & $\begin{array}{l}0 \\
1 \\
2 \\
3\end{array}$ & $\begin{array}{r}-1.880 \\
-4.740 \\
-13.205 \\
-26.560\end{array}$ & $\begin{array}{l}0 \\
1 \\
2 \\
3 \\
4\end{array}$ & $\begin{array}{r}-1.210 \\
-3.100 \\
-8.660 \\
-17.620 \\
-29.340\end{array}$ \\
\hline $6_{06} \leftarrow 5_{05}$ & $|\mathbf{M}|=\begin{array}{r}1 \\
2 \\
3 \\
4\end{array}$ & $\begin{array}{r}-11.245 \\
-9.295 \\
-6.005 \\
-1.445\end{array}$ & $\begin{array}{l}1 \\
2 \\
3 \\
4\end{array}$ & $\begin{array}{l}-7.217^{a} \\
-5.975 \\
-3.895 \\
-0.995\end{array}$ & $\begin{array}{l}1 \\
2 \\
3 \\
4\end{array}$ & $\begin{array}{l}-4.785 \\
-3.895 \\
-2.605 \\
-0.630^{a}\end{array}$ \\
\hline $6_{15} \leftarrow 5_{14}$ & 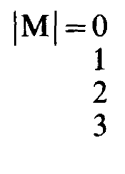 & $\begin{array}{r}-1.850 \\
-2.985 \\
-6.480 \\
-12.230\end{array}$ & $\begin{array}{l}0 \\
1 \\
2 \\
3\end{array}$ & $\begin{array}{l}-1.190 \\
-1.930 \\
-4.180 \\
-7.875\end{array}$ & $\begin{array}{l}0 \\
1 \\
2 \\
3 \\
4\end{array}$ & $\begin{array}{l}-0.815^{a} \\
-1.250 \\
-2.725 \\
-5.260 \\
-8.525\end{array}$ \\
\hline
\end{tabular}

${ }^{a}$ Sweep rate $0.02 \mathrm{MHz} / \mathrm{s}$. 
except for the constants corresponding to the motion of the fluorine atom. Those force constants were taken from Freeman et al. ${ }^{6}$ The lowest-energy vibration is a torsional mode and this was calculated to have the frequency $142 \mathrm{~cm}^{-1}$, which is in good agreement with the above experimental value. The next two out-of-plane modes were calculated to have the frequencies 262 and 602 $\mathrm{cm}^{-1}$. The three lowest in-plane fundamentals had their calculated frequencies at 222, 429 and 472 $\mathrm{cm}^{-1}$.

\section{STARK EFFECT AND DIPOLE MOMENT}

In order to determine the dipole moment of the molecule a study of the Stark effect was undertaken. The Stark lobes of $5_{05} \leftarrow 4_{04}, 5_{14} \leftarrow 4_{13}, 6_{06} \leftarrow 5_{05}$ and $6_{15} \leftarrow 5_{14}$ were measured at different electric field strengths: see Table 4. During these measurements a sweep rate of $0.05 \mathrm{MHz} / \mathrm{s}$ was usually employed. However, for lobes that were partially overlapped the lower sweep rate $0.02 \mathrm{MHz} / \mathrm{s}$ was chosen, thereby diminishing the uncertainty in the frequency measurement due to the nearby line (all non-Stark effect lines were recorded at both sweep rates).

For $5_{05} \leftarrow 4_{04}$ and $6_{06} \leftarrow 5_{05}$ the Stark effect was treated as a pure second-order effect for the electric field strengths used. However, in order to treat the Stark effect for $5_{14} \leftarrow 4_{13}$ and $6_{15} \leftarrow 5_{14}$ coupling between nearly degenerate levels was also considered. There was coupling between $5_{14}$ and $5_{15}$, between $4_{13}$ and $4_{14}$ and also between $6_{15}$ and $6_{16}$ (all by $\mu_{\mathrm{a}}$ ). For the lowest of the field strengths used, the near-coincidence in energy between $6_{15}$ and $6_{16}$ was, however, removed. Perturbation calculations based on the van Vleck transformation were performed in the usual way. ${ }^{7}$ The matrix elements of $\bar{\mu} \cdot \bar{E}$ were calculated, as described by Schwendeman, ${ }^{8}$ in a computer program written by M. Ribeaud at the Swiss Federal Institute of Technology. The program calculated the necessary coefficients for the equations that were used in a non-linear least-squares iterative computer program to determine the components of the dipole moment vector. This procedure was followed for each of the three electric field strengths: see Table 5.

One of the values of $|\bar{\mu}|$ may seem to be larger than the others. However, the error limits given for the three values of $|\bar{\mu}|$ are probably too narrow, because there is an additional uncertainty of about
Table 5. Values of the dipole moment (Debye units) obtained from a least-squares fit of the observed Stark shifts to the theoretical expressions.

\begin{tabular}{|c|c|c|c|}
\hline $\begin{array}{l}\text { Electric field } \\
\text { strength }(\mathrm{V} / \mathrm{cm})\end{array}$ & $\left|\mu_{\mathrm{a}}\right|$ & $\left|\mu_{\mathrm{b}}\right|$ & $|\bar{\mu}|^{b}$ \\
\hline $3147(3)$ & $1.212(1)$ & $0.701(1)$ & $1.400(1)$ \\
\hline $2573.1(4)$ & $1.1875(5)$ & $0.6889(10)$ & $1.3729(7)$ \\
\hline $2081.5(8)$ & $1.1802(16)$ & $0.6916(37)$ & $1.368(2)$ \\
\hline Mean value: $:^{a}$ & $1.193(17)$ & $0.694(6)$ & $1.380(17)$ \\
\hline
\end{tabular}

${ }^{a}$ The error limits are one standard deviation (see text).

${ }^{b}\left|\mu_{\mathrm{c}}\right|=0$ (see text).

$1 \%$ in the calculations that take near-degeneracy into account. The value for the dipole moment of the molecule is (with standard deviation) $1.380 \pm$ 0.017 Debye units.

It is interesting to compare the above value with values for the dipole moment of some other hydrocarbons containing one fluorine atom. ${ }^{9}$ For fluoroethene the value reported is $1.427 \pm 0.010 \mathrm{D},{ }^{10}$ whereas for trans-fluoropropene it is $1.85 \pm 0.02$ D. ${ }^{11}$ The vinyl group of trans-1-fluoro-1,3-butadiene could be said to have a noticeable electron drawing ability resulting in the somewhat smaller value $1.380 \pm 0.017 \mathrm{D}$ as compared to fluoroethene.

For accurate determination of the electric field strengths the OCS line $2 \leftarrow 1$ was used as a standard, with $0.71521 \mathrm{D}$ as the value of the dipole moment for carbonyl sulfide. Values of the physical constants necessary in the calculations of the field strengths were the same as those used in the Stark effect computer program. ${ }^{12}$

Acknowledgements. I would like to express my gratitude to Dr. Fred Karlsson for many fruitful discussions, particularly in connection with the synthesis, and for valuable comments on the manuscript. I am also very grateful to Dr. Stig Ljunggren at the Royal Institute of Technology, Stockholm, for allowing me to use the microwave spectrometer, and for providing me with the computer program dealing with the Stark effect and for valuable discussions in this context.

\section{REFERENCES}

1. Viehe, H. G. Chem. Ber. 93 (1960) 1706.

2. Viehe, H. G., Dale, J. and Franchimont, E. Chem. Ber. 97 (1964) 248.

3. Viehe, H. G. Chem. Ber. 97 (1964) 600. 
4. Gordy, W. and Cook, R. L. Microwave Molecular Spectra, Interscience, New York 1970.

5. Borg, A., Smith, Z., Gundersen, G. and Klaeboe, P. Spectrochim. Acta Part A 36 (1980) 119.

6. Freeman, J. M. and Henshall, T. Can. J. Chem. 47 (1969) 941.

7. Golden, S. and Wilson, E. B., Jr. J. Chem. Phys. 16 (1948) 669.

8. Schwendeman, R. H. J. Mol. Spectrosc. 7 (1961) 280.

9. McClellan, A. L. Tables of Experimental Dipole Moments, Freeman, San Francisco 1963.

10. Mirri, A. M., Guarnieri, A. and Favero, P. Nuovo Cimento 19 (1961) 1189.

11. Siegel, S. J. Chem. Phys. 27 (1957) 989.

12. Natl. Bur. Stand. (U.S.), Tech. News Bull. 47 (1963) No. 10, October.

Received February 8, 1980. 